\title{
Propagation in a Model Terrestrial Waveguide of Nonuniform Height: Theory and Experiment ${ }^{1}$
}

\author{
E. Bahar and James R. Wait \\ Department of Electrical Engineering, University of Colorado, Boulder, Colo.
}

(Received May 11, 1965)

\begin{abstract}
Propagation of electromagnetic waves in multimode waveguides of variable height is investigated. The model consists of two uniform rectangular waveguides connected by a linearly tapered waveguide section. Using a generalized reciprocity theorem for waveguide junctions, a previous quasi-optic solution of this problem is "extended to account for reflected waves. The results have application to the theory of VLF radio propagation when the effective height of reflection of the ionosphere boundary varies along the path. The analytical investigation has been complemented by laboratory measurements taken from a two-dimensional microwave model, and good agreement with calculated results was achieved.
\end{abstract}

\section{Introduction}

The diurnal variation of the electrical properties of the ionosphere results in a change of the effective height of the ionosphere lower boundary along the path of propagation. These spatial variations of the effect height of reflection modify the field patterns across the waveguide. If the change is sufficiently gradual the waveguide theory developed for a uniform earth-ionosphere waveguide may be generalized in a fairly straightforward manner [e.g., see Wait, 1964]. Furthermore, the observed data [Crombie, Allen, and Newman, 1958; W ait, 1959, 1961] are consistent with this picture. However, it is now apparent that near sunset and sunrise lines, mode conversion effects may be important. It is the purpose of this paper to consider this problem.

A two-dimensional dual model waveguide described previously [Bahar and Wait, 1964] is the basic tool in the present investigation. In this scaled model, rather ideal conditions are assumed. At the ionosphere boundary (considered to be sharply bounded), the tangential magnetic field of. the VLF radio wave is assumed to vanish, corresponding to a reflection coefficient $R_{i}=-1$. The earth's boundary is assumed to be perfectly conducting, corresponding to a reflection coefficient $R_{g}=1$. Consideration is restricted to the lower order modes (which account for most of the energy of the VLF radio waves at large distances from the source). It has also been assumed in this work that

$$
h / a_{0}<<\left|C^{2}\right| \text { and }\left(k a_{0} / 2\right)^{1 / 3} \operatorname{Re} C>2,
$$

where $a_{0}$ is the radius of the earth, $h$ is the effective height of the sharply bounded ionosphere, $k$ is equal to $2 \pi /$ free-space wavelength, and $C$ is the cosine of the angle of incidence at the earth's boundary. With these restrictions a flat earth approximation can be introduced. Furthermore, the effects of the earth's magnetic field have been neglected.

It has been shown [W ait, 1964; Bahar and Wait, 1964] that, with the assumptions stated above, the modal equation governing the propagation of TM modes in the actual earth-ionosphere waveguide is the same as for the TE modes in the dual model waveguide of half-height corresponding to the height of the ionosphere in wavelengths. The cosine of the angle of incidence of the $p$ th mode on the boundaries of the waveguide, derived from the appropriate modal equation [Wait,

${ }^{1}$ The research reported in this paper was sponsored by the Advanced Research Projects Agency on Contract C.S.T. 7348 (ARPA Order No. 183-62). 
1960], is given by

$$
C_{p}=\frac{\pi(2 p-1)}{2 k h}, \quad p=1,2,3
$$

where $k=2 \pi / \lambda$ is the wave number mentioned above.

The original curved earth-ionosphere waveguide is represented by the upper (or lower) half of the rectangular dual model waveguide (see fig. 1). The upper (or lower) conducting boundary of the model waveguide represents the ionosphere boundary and the plane of symmetry $(x, z)$ represents the earth boundary. Also TM modes propagating in the earth-ionosphere waveguide are represented in the dual model by TE modes. Of course, it is necessary that only waves with electric fields that are symmetric about the $x$-axis of the model waveguide are initially launched into the waveguide; thus the tangential magnetic field at the plane of $\operatorname{symmetry}(x, z)$ vanishes, which conforms with the vanishing tangential electric field at the earth's surface. It should also be noted then that the $p$ th mode in the earth-ionosphere waveguide corresponds to the $(2 p-1)$ th mode in the model waveguide since the even modes with asymmetric electric fields must be excluded. A more detailed discussion of the modeling technique is given elsewhere [Maley and Bahar, 1963, 1964; Bahar, 1964].

The diurnal change in the effective height of the ionosphere derived from phase velocity measurements as reported by Pierce [1955] and Crombie, Allen, and Newman [1958] have been discussed by Wait [1959, 1961]. From the viewpoint of mode theory of VLF propagation, Wait obtains a value of $\Delta h$ (the change in the effective height) between 16 and $18 \mathrm{~km}$, in good agreement with values obtained by Bain et al. [1952], who analyzed the interference pattern of the groundwave and the first hop skywave at $16 \mathrm{kc} / \mathrm{s}$. The model waveguide with a half-height $h_{d}=12.7 \mathrm{~cm}$ (operated at $9 \mathrm{Gc} / \mathrm{s}$ ) would thus represent the earth-ionosphere waveguide $88 \mathrm{~km}$ high (at about $13 \mathrm{kc} / \mathrm{s}$ ) under more or less normal conditions prevailing at night. Similarly the model waveguide with half-height $h_{a}=10.16 \mathrm{~cm}$ would represent the earth-ionosphere waveguide $70 \mathrm{~km}$ high under the conditions prevailing during daytime. This paper deals with a special case in which the transition between the day and night propagation paths is assumed to take place over a length of several wavelengths rather than in the abrupt manner discussed in an earlier paper by the same authors [Bahar and Wait, 1964]. The transition region is assumed to be wedge-shaped, and it is bounded by uniform waveguide sections of unequal cross sections representing the day and night propagation paths.

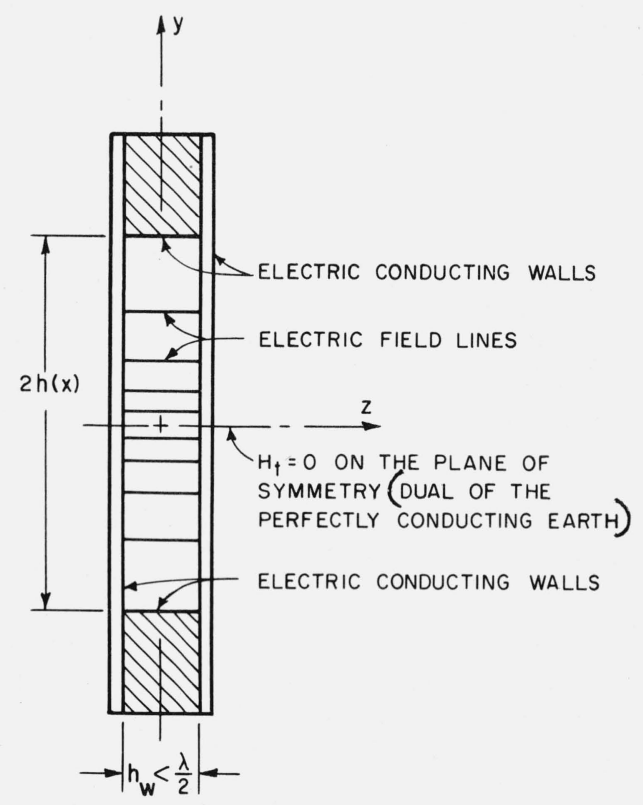

FIGURE 1. Cross section of the two-dimensional dual model of the idealized earth-ionosphere waveguide used in the experimental investigation. 


\section{Formulation of the Problem}

The half-height $h(x)$ of the waveguide, as a function of the distance $x$ along the axis, is expressed analytically by

$$
h(x)=h_{a}+[u(x)-u(x-L)] \frac{h_{d}-h_{a}}{L} x+u(x-L)\left(h_{d}-h_{a}\right)
$$

where $u(x)$ is the unit step function defined as,

$$
u(x)=\left\{\begin{array}{l}
0 \text { for } x<0 \\
1 \text { for } x \geqslant 0,
\end{array}\right.
$$

$L$ is the length of the transition path, and $h_{a}$ and $h_{d}$ are the heights of the uniform waveguide sections at the terminals of the transition region. The gradient of the height profile in the transition region is restricted in the following analysis by the condition

$$
\psi \approx \tan \psi=\frac{h_{d}-h_{a}}{L}<0.25
$$

The total variation in height is assumed to be about a wavelength and, thus, the length of the transition region is restricted by

$$
L>4\left(h_{d}-h_{a}\right) \approx 4 \lambda \text {. }
$$

This is not a serious restriction judging from the nature of the problem under consideration.

The linear transition described here has been investigated by Wait [1962], who considered a TM wave incident on a wedge-shaped transition between two parallel-plate waveguides. These solutions are extended in this paper. Here the transition region between the uniform waveguides is analyzed as a waveguide junction.

To derive the scattering of the waveguide modes for the case of propagation in the day-tonight path, a quasi-optical method is used in which reflections are totally neglected. This approximation is very suitable whenever a low-order mode propagates through a multimode waveguide in the direction of increasing cross section, as was illustrated rather rigorously in the investigation of mode conversion for the case of propagation of grazing modes in a multimode waveguide with an abrupt height discontinuity [Bahar and Wait, 1964]. The above solution is then used to derive the scattering when the direction of propagation is reversed (night-to-day path) by invoking the reciprocity theorem. In this manner, reflections for the night-to-day propagation path need not be neglected.

In order to apply the reciprocity condition at each discontinuity in the gradient of the height profile ( $x=0, x=L$ in fig. 2 ), it is necessary to derive a generalized interpretation of the reciprocity theorem for waveguide junctions.

FIGURE 2. Wedge-shaped transition region between the day and night propagation paths.

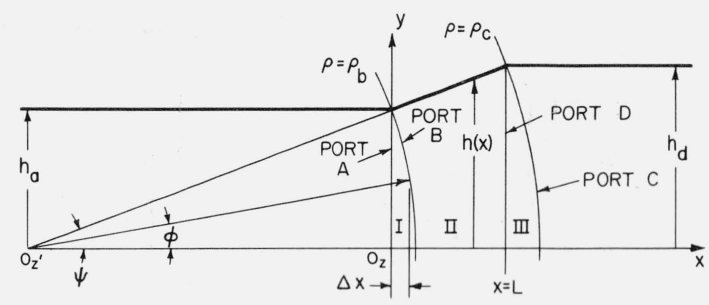


At the terminal surfaces in the wedge region (ports A, B, C, and D in fig. 2), the tangential fields are described by a finite number of unattenuated modes and an infinite number of evanescent modes. The electric "basis" field (transverse field pattern) for the $n$th mode in ports A and B, for instance, are denoted by $\Phi_{n}^{A}$ and $\Phi_{n}^{B}$ respectively. In the problem under consideration

$$
\Phi_{n}^{A}=\cos k_{n}^{A} y_{a}, \quad|y| \leqslant h_{a} \text { and } \Phi_{n}^{B}=\cos \nu_{n} \varphi, \quad|\varphi| \leqslant \psi
$$

where

$$
k_{n}^{A}=\frac{n \pi}{2 h_{a}}
$$

and

$$
\nu_{n}=\frac{n \pi}{2 \psi}
$$

It will be found very convenient to use matrix notation throughout this work and thus, before proceeding with the analysis, the definitions of the symbols for the matrices and their elements are stated.

Let $\Phi^{A}$ and $\Phi^{B}$ denote the "basis" field row vectors whose elements are $\Phi_{n}^{A}$ and $\Phi_{n}^{B}$ respectively, and let $\Phi$ denote the total "basis" fields row vector, representing all the "basis" fields in both the ports $\mathrm{A}$ and $\mathrm{B}$, of the junction under consideration. Hence $\Phi$ is defined as

$$
\Phi=\left[\Phi^{A} \Phi^{B}\right] .
$$

The quantities $a_{n}^{A}$ and $b_{n}^{A}$ are defined as the $n$th mode complex wave amplitude (referred to port A) of the wave traveling towards and away from the waveguide junction respectively. Thus $\left|a_{n}^{A}\right|$ is the magnitude of the forward traveling $n$th mode and $\arg \left(a_{n}^{A}\right)$ determines the phase of this wave relative to the other waveguide modes. The symbols $a^{A}$ and $b^{A}$ are wave-amplitude column vectors whose elements are $a_{n}^{A}$ and $b_{n}^{A}$ respectively. Similarly, with respect to terminal $\mathrm{B}, a^{B}$ and and $b^{B}$ are wave-amplitude column vectors whose elements are $a_{n}^{B}$ and $b_{n}^{B}$ respectively. The total wave-amplitude column vectors are defined as

$$
a=\left[\begin{array}{c}
a^{A} \\
a^{B}
\end{array}\right] \text { and } b=\left[\begin{array}{c}
b^{A} \\
b^{B}
\end{array}\right]
$$

Then the electric field at port A expressed in terms of the "basis" fields and wave amplitudes is

$$
E_{Z}^{A}(y, t)=\operatorname{Re}\left\{\Phi^{A}\left[a^{A}+b^{A}\right] \exp (i w t)\right\} .
$$

Matrices $Y^{A}$ and $Y^{B}$ are diagonal characteristic admittance matrices whose elements $Y_{n}^{A}$ and $Y_{n}^{B}$ are the $n$th mode characteristic admittances for ports $\mathrm{A}$ and $\mathrm{B}$ respectively. The total characteristic wave admittance is defined as

$$
Y=\left[\begin{array}{cc}
Y^{A} & 0 \\
0 & Y^{B}
\end{array}\right] .
$$


The symbols $Z^{A}, Z^{B}$, and $Z$ are characteristic impedance matrices. They are equal to the inverse of the matrices $Y^{A}, Y^{B}$, and $Y$ respectively.

Matrices $W^{A}$ and $W^{B}$ are diagonal matrices whose elements $W_{n}^{A}$ and $W_{n}^{B}$ are the power normalization factors defined by

$$
W_{n}^{A}=\int_{-h_{a}}^{h_{a}}\left[\Phi_{n}^{A}\right]^{2} d y=h_{a} \text { and } W_{n}^{B}=\int_{-\psi}^{\psi}\left[\Phi_{n}^{B}\right]^{2} \rho d \varphi=\rho_{b} \psi
$$

The total power normalization matrix is defined as

$$
W=\left[\begin{array}{cc}
W^{A} & 0 \\
0 & W^{B}
\end{array}\right]=\left[\begin{array}{cc}
h_{a} & 0 \\
0 & \rho_{b} \psi
\end{array}\right],
$$

where $I$ is the unit diagonal matrix.

The symbol $S^{A}$ is a diagonal matrix whose elements are $S_{n}^{A} ; S_{n}^{A}$ can be identified as the sine of the angle of incidence of the $n$th mode on the narrow walls of the model waveguide in port A. It is given by

$$
S_{n}^{A}=\left[1-\left(k_{n}^{A} / k\right)^{2}\right]^{1 / 2}, \quad n=1,3,5 \ldots
$$

where $k_{n}^{A}$ is given by (6a). These elements are related to the mode characteristic admittance through the equation

$$
Y_{n}^{A}=Y S_{n}^{A},
$$

where $Y$ is the free-space wave admittance. They are also related to the mode propagation constants $\beta_{n}^{A}$ through the equation

$$
\beta_{n}^{A}=k S_{n}^{A},
$$

where $k$ is the free-space wave number.

Symbol $S^{A A}$ is a square reflection scattering matrix, whose element $S_{n m}^{A A}$ is the complex amplitude of the $n$th reflected mode when the $m$ th mode of unit amplitude is incident on the junction from port A. Similarly, $S^{B B}$ is the reflection scattering matrix related to the waves in port B.

The symbol $S^{B A}$ is a square transmission scattering matrix, whose element $S_{n m}^{B A}$ is the complex amplitude of the $n$th mode transmitted through the junction to port $\mathrm{B}$ when the $m$ th mode of unit amplitude is incident on the junction from port A. Similarly $S^{A B}$ is the transmission scattering matrix related to transmission through the junction from port B to port A.

The total scattering matrix is then defined as

$$
S=\left[\begin{array}{ll}
S^{A A} & S^{A B} \\
S^{B A} & S^{B B}
\end{array}\right] .
$$

In terms of the matrices defined above, the scattering matrix equation of the waveguide junction becomes

$$
b=S a \text {. }
$$

Similarly, the above matrix quantities can be defined corresponding to ports C and D (fig. 2). 


\section{List of Symbols}

$a, a^{A}=$ forward traveling wave amplitude vectors

$\mathrm{a}_{0}=$ radius of the earth

$b, b^{A}=$ backward traveling wave amplitude vector

$h=$ height of the ionosphere, or half-height of model waveguide

$h_{a}=$ half-height of "daytime" portion of waveguide

$h_{d}=$ half-height of "nighttime" portion of waveguide

$i=\sqrt{-1}$

$k=$ free-space wave number

$m, n, p, q, s=$ integer indices

$u=$ unit step function

$x, y, z=$ Cartesian coordinates

$E=$ electric field

$\hat{E}=$ electric field amplitude matrix

$H=$ magnetic field

$\hat{H}=$ magnetic field amplitude matrix

$R=$ reflection coefficient

$S=$ scattering matrix

$S^{A A}=$ reflection scattering matrix

$S^{B A}=$ transmission scattering matrix

$S_{m}^{A}=$ sine of the angle of incidence for the $m$ th mode

$W=$ power-normalization matrix

$Y=$ free-space wave admittance, characteristic admittance matrix

$\hat{Y}=$ equivalent admittance matrix

$Z=$ characteristic impedance matrix

$\beta=$ propagation constant

$\epsilon=$ dielectric constant

$\lambda=$ free-space wavelength

$\mu=$ permeability

$\Phi, \Phi^{A}=$ basis-field row vector

$\rho, \varphi, z^{\prime}=$ cylindrical coordinates.

\section{Day-to-Night Transition Path}

The incident wave traveling in the direction of the axis (from the narrow to the wide uniform waveguide as indicated in fig. 2) is assumed to be the $\mathrm{TE}_{m, 0}$ mode for which the electric field in the narrow waveguide is given by

$$
E_{z}=a_{m}^{A} \exp \left(-i k x S_{m}^{A}\right) \Phi_{m}^{A}(y)=a_{m}^{A} \exp \left\{-i k x S_{m}^{A}\right\} \cos k_{m}^{A} y
$$

where $k_{m}^{A}$ and $S_{m}^{A}$ are defined by (6) and (12) respectively.

For the case considered, the width of the model waveguide increases monotonically (or remains constant) as the wave advances along the waveguide, and the cross section of the multimode waveguide never changes abruptly. Thus, the assumption that reflections may be neglected can be applied; this renders $S^{A A}=0, S^{C C}=0$ (actually this assumption was also verified experimentally).

The waveguide region shown in figure 2 is regarded to possess junctions for the sake of analysis. For example:

Junction I is bounded by the $x=0$ plane and the $\rho=\rho_{b}$ cylindrical surface,

Junction II is bounded by the cylindrical surfaces $\rho=\rho_{b}$ and $\rho=\rho_{c}$, and

Junction III is bounded by the cylindrical surface $\rho=\rho_{c}$ and the plane $x=L$. 
Junction II, being a uniform linear wedge region, will transmit the incident waves without mode conversion.

(a) Scattering through Junction I.

Let $x, y, z$ be the coordinate system connected with port $\mathrm{A}\left(x=0\right.$ plane) and let $\rho, \varphi, z^{\prime}$ be the coordinate system connected with port B $\left(\rho=\rho_{b}\right.$ cylindrical surface). The axes $z$ and $z^{\prime}$ are parallel as indicated in figure 2 .

The distance $\Delta x$ (along the $x$-axis) between the terminal surfaces $x=0$ and $\rho=\rho_{b}$ of junction I, as a function of the azimuthal angle $\varphi$, is

$$
\Delta x=\rho_{b}(\cos \varphi-\cos \psi) \simeq \frac{\rho_{b}}{2}\left(\psi^{2}-\varphi^{2}\right)
$$

where $\psi$ is given by (3) and $\cos \psi$ is replaced by the first two terms of its Maclaurin series expansion. The radius $\rho_{b}$ is related to the parameters of the height profile (2) by the equation

$$
\rho_{b}=\frac{h_{a}}{\sin \psi} \approx \frac{h_{a}}{\psi}
$$

with a similar relationship for $\rho_{c}$.

On neglecting reflections, the electric field at $\rho=\rho_{b}$, by substituting (18) into (17), is given by

$$
E_{z}\left(\rho=\rho_{b}\right)=a_{m}^{A} \exp \left\{-i k S_{m}^{A} \Delta x\right\} \Phi_{m}^{A} \approx a_{m}^{A} \exp \left\{-i k \rho_{b} S_{m}^{A} \psi^{2} / 2\right\} \cdot \exp \left\{i k \rho_{b} S_{m}^{A} \varphi^{2} / 2\right\} \cos k_{m}^{A} y .
$$

The electric field in a uniform radial waveguide is expressed in terms of the orthogonal basis fields $\Phi^{B}$ by the equation

$$
E_{z}=\sum_{n} b_{n}^{B} \frac{H_{\nu_{n}}^{(2)}(k \rho)}{H_{\nu_{n}}^{(2)}\left(k \rho_{b}\right)} \Phi_{n}^{B}
$$

where $\Phi_{n}^{B}$ is given by (5), and $H_{\nu_{n}}^{(2)}$ is the Hankel function of the second kind and order $\nu_{n}$. The form of (21) follows immediately from an earlier analysis of the wedge region [Wait, 1962].

It now immediately follows that the continuity condition of the electric field at $\rho=\rho_{b}$ is given by

$$
a_{m}^{A} \exp \left\{-i k \rho_{b} S_{m}^{A} \psi^{2} / 2\right\} \exp \left\{i k \rho_{b} S_{m}^{A} \varphi^{2} / 2\right\} \Phi_{m}^{A} \equiv \sum_{n} \Phi_{n}^{B} b_{n}^{B} \equiv \sum_{n} \Phi_{n}^{B} S_{n m}^{B A} a_{m}^{A}
$$

Now, subject to the restriction of (3) on the gradient of the height profile,

$$
k_{m}^{A} y=\frac{m \pi y}{2 h_{a}}=\frac{m \pi \sin \varphi}{2 \sin \psi} \approx \frac{m \pi \varphi}{2 \psi}=\nu_{m} \varphi .
$$

To solve for the scattering coefficients $S_{n m}^{B A}$, premultiply by $\Phi_{n}^{B}$ and integrate with respect to $\varphi$ over the interval $(0, \psi)$; thus

$$
\begin{aligned}
S_{n m}^{B A}=\frac{2}{\psi} \exp \left\{-i k \rho_{b} S_{m}^{A} \psi^{2} / 2\right\} & \\
\times \int_{0}^{\psi} \exp \left\{i k \rho_{b} S_{m}^{A} \varphi^{2} / 2\right\} \cos \nu_{n} \varphi \cos \nu_{m} \varphi d \varphi & =\frac{2}{\psi} \exp \left\{-i k \rho_{b} S_{m}^{A} \psi^{2} / 2\right\} I_{n m}^{B A} .
\end{aligned}
$$


As indicated before [Wait, 1962], the integral $I_{n m}^{B A}$ in the above equation can be expressed in terms of a Fresnel integral $F(x)$ defined by

$$
F(x)=\int_{0}^{x} \exp \left\{\frac{i \pi x^{2}}{2}\right\} d x
$$

To be explicit,

$$
\begin{aligned}
& I_{n m}^{B A}=\frac{1}{4}\left(\frac{\pi}{2 \alpha_{m}^{A}}\right)^{1 / 2}\left\{\operatorname { e x p } [ \frac { - i ( \nu _ { m } - \nu _ { n } ) ^ { 2 } } { 4 \alpha _ { m } ^ { A } } ] \left[F\left\{\left(\frac{2 \alpha_{m}^{A}}{\pi}\right)^{1 / 2}\left(\psi+\frac{\nu_{m}-\nu_{n}}{2 \alpha_{m}^{A}}\right)\right\}\right.\right. \\
& \left.+F\left\{\left(\frac{2 \alpha_{m}^{A}}{\pi}\right)^{1 / 2}\left(\psi-\frac{\nu_{m}-\nu_{n}}{2 \alpha_{m}^{A}}\right)\right\}\right] \\
& \left.+\exp \left[\frac{-i\left(\nu_{m}+\nu_{n}\right)^{2}}{4 \alpha_{m}^{A}}\right]\left[F\left\{\left(\frac{2 \alpha_{m}^{A}}{\pi}\right)^{1 / 2}\left(\psi+\frac{\nu_{m}+\nu_{n}}{2 \alpha_{m}^{A}}\right)\right\}+F\left\{\left(\frac{2 \alpha_{m}^{A}}{\pi}\right)^{1 / 2}\left(\psi-\frac{\nu_{m}+\nu_{n}}{2 \alpha_{m}^{A}}\right)\right\}\right]\right\}
\end{aligned}
$$

where

$$
\alpha_{m}^{A}=\frac{k \rho_{b} S_{m}^{A}}{2}
$$

(b) Transmission through the uniform radial waveguide Junction II.

In this junction no scattering takes place, and it can readily be shown [Wait, 1962] that the transmission coefficients in the forward and backward directions between the terminal surfaces $\rho_{b}$ and $\rho_{c}$ are given, respectively, by

$$
\begin{gathered}
S_{m n}^{C B}=H_{\nu_{m}}^{(2)}\left(k \rho_{c}\right) / H_{\nu_{m}}^{(2)}\left(k \rho_{b}\right) \delta_{m n} \\
S_{m n}^{B C}=H_{\nu_{m}}^{(1)}\left(k \rho_{b}\right) / H_{\nu_{m}}^{(1)}\left(k \rho_{c}\right) \delta_{m n} .
\end{gathered}
$$

The relationship between the coefficients $S_{n m}^{C B}$ and $S_{n m}^{B C}$ checks with the general reciprocity theorem (appendix A) applied to uniform radial waveguides. The $H_{\nu_{m}}^{(1)}$ and $H_{\nu_{m}}^{(2)}$ are the Hankel functions of the first and second kind (of order $\nu_{m}$ ) respectively.

(c) Scattering through Junction III.

One should appreciate that the wave incident on Junction III comprises several modes even when only one mode is incident on Junction I. However, in the analysis it is necessary to consider only one of these modes incident on Junction III. On using superposition the result for several incident modes is readily obtained. The incident wave approaching Junction III from the radial waveguide is thus taken to be

$$
E_{z}=a_{m}^{C} \frac{H_{\nu_{m}}^{(2)}(k \rho)}{H_{\nu_{m}}^{(2)}\left(k \rho_{c}\right)} \Phi_{m}^{C}
$$

where $\Phi_{m}^{C}=\Phi_{m}^{B}$ is given by (5).

The basis fields in the rectangular waveguide are

$$
\Phi_{m}^{D}=\cos k_{n}^{D} y
$$


where

$$
k_{n}^{D}=\frac{n \pi}{2 h_{d}}
$$

The electric field in the rectangular waveguide expressed in terms of these basis fields is

where

$$
E_{z}=\sum_{n} b_{n}^{D} \exp \left[-i k(x-L) S_{n}^{D}\right]
$$

$$
S_{m}^{D}=\left[1-\left(k_{m}^{D} / k\right)^{2}\right]^{1 / 2}
$$

Neglecting reflections, and following the analysis applied to Junction I, it readily follows that the continuity condition for the electric field at $x=L$ is given by

$$
a_{m}^{C} \exp \left\{i k \rho_{c} S_{m}^{C} \psi^{2} / 2\right\} \exp \left\{-i k \rho_{c} S_{m}^{C} \varphi^{2} / 2\right\} \equiv \sum_{n} \Phi_{n}^{D} b_{n}^{D} \equiv \sum_{n} \Phi_{n}^{D} S_{n m}^{D C} a_{m}^{C}
$$

in which $S_{m}^{C}=S_{m}^{D}$.

To solve for $S_{n m}^{D C}$, premultiply by $\Phi_{m}^{D}$ and integrate with respect to $y$ over the interval $\left[0, h_{d}\right]$. This gives

$$
\begin{aligned}
S_{n m}^{D C}=\exp \left\{i k \rho_{c} S_{m}^{C} \psi^{2} / 2\right\} \frac{2}{d} \int_{0}^{h_{d}} \exp \left\{-i k \rho_{c} S_{m}^{C} \varphi^{2} / 2\right\} \cos \nu_{m} \varphi \cos k_{n}^{D} y d y & \\
\approx \exp \left\{i k \rho_{c} S_{m}^{C} \psi^{2} / 2\right\} \frac{2}{\psi} \int_{0}^{\psi} \exp \left\{-i k \rho_{c} S_{m}^{C} \varphi^{2} / 2\right\} & \cos \nu_{m} \varphi \cos \nu_{n} \varphi d \varphi \\
& =\exp \left\{i k \rho_{c} S_{m}^{C} \psi^{2} / 2\right\} \frac{2}{\psi}\left(I_{n m}^{D C}\right)^{*},
\end{aligned}
$$

where, as in (23), it is assumed that

$$
k_{n}^{D} y \approx \nu_{n} \varphi \text { and } \frac{d y}{h_{d}} \approx \frac{d \varphi}{\psi}
$$

and $\left(I_{n m}^{D C}\right)^{*}$ is the complex conjugate of $I_{n m}^{D C}$. The latter is defined by

$$
I_{n m}^{D C}=\int_{0}^{\psi} \exp \left\{i \alpha_{m}^{C} \varphi^{2}\right\} \cos \nu_{n} \varphi \cos \nu_{m} \varphi d \varphi
$$

which in turn can be expressed in terms of the Fresnel integrals as in (26). Thus

$$
\alpha_{m}^{C}=\frac{k \rho_{c} S_{m}^{C}}{2}
$$

Combining the results of the scattering and transmission in the separate sections of the wedge region and denoting the scattering coefficients of the composite Junction (with terminal planes at $x=0$ and $x=L$ ) $T$, it can be readily shown that the transmission scattering matrix (from port A to port $\mathrm{D}$ ) is given by

$$
T^{D A}=S^{D C} S^{C B} S^{B A}
$$


where $S^{B A} S^{C B}$ and $S^{D C}$ are given by (24), (28), and (36). The reflection scattering matrix for port A is assumed to be the zero matrix

$$
T^{A A}=0
$$

The electric field at $x=L$ is therefore given by the matrix multiplication

$$
E_{z}(x=L)=\Phi^{D} T^{D A} a^{A} .
$$

\section{Night-to-Day Tapered Transition Path}

When the cross section of the model waveguide decreases as the incident wave propagates in a nonuniform waveguide (propagation in the direction of decreasing $\rho$ or $x$ ), the reflections cannot be considered negligible unless the gradient of the effective height profile is very small. As in the case when the height profile had an abrupt discontinuity [Bahar and Wait, 1964], it was observed experimentally that there were substantial reflections in the case of a wedge transition region (for the night-to-day transition path), even when the gradient (3) of the height profile was $\psi \simeq \tan \psi$ $=0.25$. In view of this, the method developed in the previous section to derive the scattering matrices is not effective in this case. However, since a very good approximation to the solution of scattering in the day-to-night propagation path is already available from the preceding analysis, the solution to the problem of scattering in the night-to-day path can be obtained readily from it by the use of the reciprocity theorem. The reciprocity theorem, for waveguide junctions with uniform waveguide ports of constant cross section and a linear axis of propagation, is given by the matrix equation

$$
W Y S=\widetilde{W Y S}
$$

where the matrices $W Y$ and $S$ are defined in section 2 and the curled symbol above the matrices represents the transpose operation. Equation (43) states that the matrix product $W Y S$ is symmetric. The corresponding relationship between the transmission scattering matrices $S^{A B}$ and $S^{B A}$ is

$$
W^{A} Y^{A} S^{A B}=\widetilde{S^{B A}} Y^{B} W^{B}
$$

In terms of the elements of the matrices, the reciprocity theorem is given by

$$
W_{m}^{A} Y_{m}^{A} S_{m n}^{A B}=W_{n}^{B} Y_{n}^{B} S_{n m}^{B A}
$$

The derivation of the above theorem is given by Kerns [1961]. A generalization of this theorem for the case when the cross section of the terminal ports of the waveguide is not constant is given in appendix A. The scattering matrix $S^{A B}$ can therefore be obtained directly from the previous solution for $S^{B A}$ without involving any further approximations, since the reciprocity theorem is exact. Hence

$$
S^{A B}=\left[W^{A} Y^{A}\right]^{-1} S^{B A} Y^{B} W^{B}
$$

The fields transmitted through the wedge region to port A can be derived in a straightforward manner by means of the scattering matrix $T^{A D}$ in the following matrix equation:

$$
E_{z}(x=0)=\Phi^{A} T^{A D} a^{D}
$$

where the transmission scattering matrix $T^{A D}$ is related to the matrix $T^{D A}$, given by (40), through 
the reciprocity theorem as in (46),

$$
T^{A D}=\left[W^{A} Y^{A}\right]^{-1} T^{D A} Y^{D} W^{D}=\frac{b}{a} Z^{A} T^{D A} Y^{D}
$$

where the power normalization matrices $W^{A}$ and $W^{D}$ and the impedance and admittance matrices $Z^{A}, Y^{A}, Y^{D}$ are defined in section 2 .

In order to derive the fields within the wedge region or to obtain a first-order approximation of the reflection scattering matrix $T^{D D}$, it is necessary to apply the generalized reciprocity theorem (appendix A) to each of the subsections of the wedge region, (since ports B and $\mathrm{C}$ are not rectangular). In particular it is necessary to determine the scattering matrices $S^{A B}, S^{C D}, S^{B B}$, and $S^{D D}$. This shall be done in the remainder of this section.

Using the generalized reciprocity theorem, it can be readily shown that the transmission scattering coefficients through Junction I (fig. 2) from the terminal surface $\rho=\rho_{b}$ to the terminal surface $x=0$ is given by

$$
S_{m n}^{A B}=\frac{W_{n}^{B} Y_{n}^{T B}}{W_{m}^{A} Y_{m}^{T A}} S_{n m}^{B A}=\frac{S_{n m}^{B A}}{S_{m}^{A}\left(\frac{\pi k \rho_{b}}{2}\right)\left|H_{\nu_{n}}^{(1)}\left(k \rho_{b}\right)\right|^{2}}
$$

where $Y_{m}^{T A}$ and $Y_{n}^{T B}$ are the total wave admittances defined by (A.10) and (A.11) for the uniform rectangular waveguide $A$ and the uniform radial waveguide $B$ respectively. The power normalization constants defined by (10) are

$$
W_{m}^{A}=h_{a} \approx W_{n}^{B}=\rho_{b} \psi
$$

since

$$
\rho_{b} \approx a / \psi
$$

Assuming the incident field at the terminal $\rho=\rho_{b}$ of Junction I is given by $\Phi_{m}^{B} a_{m}^{B}$, the transmitted field at the plane terminal $x=0$ is

$$
E_{z}(x=0)=\sum_{n} \Phi_{n}^{A} b_{n}^{A}=\sum_{n} \Phi_{n}^{A} S_{n m}^{A B} a_{m}^{B}
$$

The total field (including reflections) at the surface $\rho=\rho_{b}$ is approximately

$$
E\left(\rho=\rho_{b}\right)=\sum_{n} \Phi_{n}^{A} S_{n m}^{A B} \exp \left\{i k S_{n}^{A} \Delta x\right\} a_{m}^{B}
$$

This field can be expressed in terms of the incident and reflected modes at the terminal surface $\rho=\rho_{b}$ by the equation

$$
E\left(\rho=\rho_{b}\right)=\sum_{p} \Phi_{p}^{B}\left[\delta_{p m}+S_{p m}^{B B}\right] a_{m}^{\ddot{B}}
$$

where $S_{p m}^{B B}$ is the reflected $p$ th mode due to an incident $m$ th mode at the terminal surface $\rho=\rho_{b}$. Hence, the continuity condition on the electric field yields

$$
\sum_{p} \Phi_{p}^{B}\left[\delta_{p m}+S_{p m}^{B B}\right] a_{m}^{B} \approx \sum_{n} \Phi_{n}^{A} S_{n m}^{A B} \exp \left\{i k S_{n}^{A} \Delta x\right\} a_{m}^{B}
$$


To determine the reflection coefficients $S_{p m}^{B B}$, premultiply by $\Phi_{p}^{B}$ and integrate with respect to $\varphi$ :

$$
\begin{gathered}
S_{p m}^{B B}+\delta_{p m} \approx \frac{2}{\psi} \sum_{n} \exp \left\{i \alpha_{n}^{A} \psi^{2}\right\}\left\{\int_{0}^{\psi} \exp \left\{-i \alpha_{n}^{A} \varphi^{2}\right\} \cos \nu_{p} \cos \nu_{n} \varphi d \varphi\right\} S_{n m}^{A B} \\
=\sum_{n} \frac{2}{\psi} \exp \left\{i \alpha_{n}^{A} \psi^{2}\right\} \overline{I_{p n}^{B A}} S_{n m}^{A B} \\
=\sum_{n}\left(S_{p n}^{B A}\right)^{*} S_{n m}^{A B}
\end{gathered}
$$

where $\left(S_{p n}^{B A}\right)^{*}$ is the complex conjugate of $S_{p n}^{B A}$ which is given by (24).

Hence, in matrix notation, the reflection scattering matrix is given by

$$
S^{B B} \approx\left(S^{B A}\right) * S^{A B}-I .
$$

The scattering matrix $S^{C D}$ for the waves that are transmitted through Junction III (in the direction of decreasing $\rho$ ), and the reflection scattering matrix $S^{D D}$ at the terminal $x=L$, are derived in the same manner as were $S^{A B}$ and $S^{B B}$, by the use of the reciprocity theorem and the continuity condition of the electric field. It can be verified that

$$
S_{m n}^{C D}=\frac{W_{n}^{D}}{W_{m}^{C}} \frac{Y_{n}^{T D}}{Y_{m}^{T C}} S_{n m}^{D C}=S_{n}^{D} \frac{\left(\pi k \rho_{c}\right)}{2}\left|H_{\nu_{m}}^{(1)}\left(k \rho_{c}\right)\right|^{2} S_{n m}^{D C}
$$

and

$$
S^{D D} \approx\left(S^{D C}\right) * S^{C D}-I
$$

where (57) and (58) are analogous to (49) and (56) respectively.

The reflection scattering matrix $T^{D D}$ for the composite junction (the entire wedge region) can now be obtained either by cascading the scattering matrices of the three elementary junctions or by direct inspection:

$$
T^{D D}=S^{D D}+S^{D C} S^{C B} S^{B B} S^{B C} S^{C D},
$$

where the first term represents the reflections due to the discontinuity in the gradient of the height profile at $x=L$, and the second term represents the reflections due to the discontinuity in the gradient at $x=0$.

The field at any point within the wedge region may be derived in a similar manner, since all the scattering coefficients have now been derived.

\section{Propagation Through a Wedge Region With a Very Small Gradient in the Height Profile}

Experimental data obtained from the model waveguide show that the reflection coefficients for either direction of propagation can be entirely neglected when the gradient of the height profile in the wedge region is restricted by the condition

$$
\psi \approx \tan \psi=\frac{h_{d}-h_{a}}{L} \leqslant 0.03
$$


Hence, for the case $\left(h_{d}-h_{a}\right)=0.76 \lambda, L$ must be about $25 \lambda$ before the reflection coefficients $T_{m n}^{D D}$ can be neglected together with the reflection coefficients $T_{m n}^{A A}$.

Provided that the condition on the gradient (60) is satisfied, it is also seen that

$$
k \frac{\rho_{b} \psi^{2}}{2} \approx \frac{\pi h_{a} \psi}{\lambda}<0.3
$$

Hence, a further simplification in the computation of the scattering coefficients can be made. The exponent in the integral of (24) can be substituted approximately by the first two terms in the Maclaurin series expansion such that, for the propagating modes $\left(S_{m}^{A}<1\right)$,

$$
\exp \left\{i \alpha_{m}^{A} \varphi^{2}\right\}=\exp \left\{\frac{i k \rho_{b} S_{m}^{A} \varphi^{2}}{2}\right\} \approx 1+\frac{i k S_{m}^{A} \rho_{b} \varphi^{2}}{2}
$$

The scattering coefficients can now be evaluated in closed form [Wait, 1962] for either direction of propagation, since reflections are now neglected in both directions. With the $m$ th mode approaching the junction from the narrow waveguide (port A), the scattering coefficients can be shown to be given by

$$
\begin{aligned}
& S_{n m}^{B A}=\exp \left\{-i k \rho_{b} S_{m}^{A} \psi^{2} / 2\right\} \\
& \simeq \exp \left\{\frac{-i k h_{a} S_{m}^{A} \psi^{2}}{2}\right\}=\left\{\begin{array}{l}
{\left[\frac{1+i k \rho_{b} S_{m}^{A} \psi^{2}}{2}\left(\frac{1}{3}-\frac{2}{\pi^{2} m^{2}}\right)\right] ; m=n} \\
\frac{i k \rho_{b} S_{m}^{A} \psi^{2}}{\pi^{2}} \frac{6 n m}{\left(m^{2}-n^{2}\right)^{2}}(-1)^{\frac{n-m}{2}} ; m \neq n \\
\exp \left[\frac{\mathrm{i} \pi h_{a} S_{m}^{A}\left(h_{d}-h_{a}\right)}{2 L \lambda}\left(\frac{1}{3}-\frac{2}{\pi^{2} m^{2}}\right)\right] ; m=n
\end{array}\right. \\
& \frac{i h_{a} S_{m}^{A}\left(h_{d}-h_{a}\right)}{\pi L \lambda} \frac{32 n m}{\left(m^{2}-n^{2}\right)^{2}}(-1)^{\frac{n-m}{2}} ; m \neq n .
\end{aligned}
$$

In the expression for $S_{11}^{B A}$ the following approximation has been introduced:

$$
1+\frac{i k h_{a} S_{m}^{A} \psi}{2}\left(\frac{1}{3}-\frac{2}{\pi^{2} m^{2}}\right) \approx \exp \left\{\frac{i k h_{a} S_{m}^{A} \psi}{2}\left(\frac{1}{3}-\frac{2}{\pi^{2} m^{2}}\right)\right\}
$$

since it has been assumed that

$$
\frac{k \rho_{b} S_{m}^{A} \psi^{2}}{2} \approx \frac{k h_{a} S_{m}^{A} \psi}{2}<<1
$$

and

$$
\psi \approx \tan \psi=\frac{h_{d}-h_{a}}{L}
$$

From this expression it is clear that the incident mode essentially undergoes a phase shift on propagating through Junction I. In the earlier analysis by Wait [1962], this phase factor was neglected.

From the above results it is also seen that, for $n \neq 1$, the scattering coefficients decrease rapidly as $\frac{1}{n^{3}}$ and that very little energy is converted into the higher order modes. It is also seen that the magnitude of the scattering coefficients is inversely proportional to the length of 
the taper region (the scattered power is inversely proportional to the square of the length of the wedge region [Solymar, 1959]).

Examination of (49) and (56) shows that, provided the condition (61) is satisfied, $S_{n m}^{B B}$ can be neglected, since not only are the reflection coefficients $S_{n m}^{A A}$ negligible, but even the transmission coefficients $S_{n m}^{B A}(n \neq m)$ are small in magnitude.

Finally, it should be pointed out that the results of the analysis in this section should be restricted to the cases when the incident wave can be assumed to be essentially comprised of lower order modes.

\section{Experimental Results}

Calculations have been carried out for the case of the principal mode propagating across the night-to-day path with $h_{a}=3.05 \lambda$ and $h_{d}=3.81 \lambda$ (fig. 2) for the following cases:
(a) $L=20 \lambda$
(b) $L=10 \lambda$.

The transverse electric field variations (amplitude and phase) obtained from the calculated scattering coefficients are plotted in figures 3 and 4, together with the experimental data from the model waveguide. The scattering coefficients $T_{n 1}^{A D}$ are tabulated in tables 1 and 2.

For the case $L=20 \lambda$, the approximate formulas (63) for the evaluation of the scattering coefficients between the rectangular and radial sections have proved to be quite accurate. [Equation (48) has been used to obtain the scattering coefficient of the entire junction. This involves the application of the reciprocity theorem so that reflections are not neglected for the night-to-day path.]

It should be noted that even in this case where the gradient of the height profile is very small, the amplitude of the third mode is approximately 12 percent of the principal mode in the narrow waveguide section. The amplitude of the higher-order modes $m \geqq 5$ drops substantially (the amplitude of the fifth mode is about 2 percent of the principal mode). This indicates that for the case of nonperfectly conducting boundaries a surface impedance concept would be applicable since most of the incident wave is scattered into near grazing modes.

From the computed data it is obvious that for the case $L=10 \lambda$ the approximate formulas for the scattering coefficients (63) are not appropriate and the more accurate formulas (24) involving the Fresnel integrals should be used. Special care should be taken in evaluating the transmission coefficients in the tapered region (28), (29) since the values of the order and the argument of the Hankel functions cross over, with the order being less than the argument for the near grazing modes and the argument being less than the order for the higher order modes. In all the above calculations only the first six (even) modes have been accounted for $(n=1,3,5,7,9,11)$, this being justified by the fact that most of the incident energy is scattered into the lower order modes.

It is rather revealing to investigate in some more detail the coefficients that are involved in the calculation of the amplitude of the third mode transmitted into the day path with the principal mode of unit amplitude incident from the night path. This is obtained by the following summation:

$$
T_{31}^{A D}=\sum_{p}^{N} S_{2 p}^{A B} S_{p p}^{B C} S_{p 1}^{C D} .
$$

Since it has been noted that only the near grazing modes are of particular significance, in order to simplify the following discussion only the first and third mode will be considered; hence

$$
T_{31}^{A D} \approx S_{33}^{A B} S_{33}^{B C} S_{31}^{C D}+S_{31}^{A B} S_{11}^{B C} S_{11}^{C D}
$$



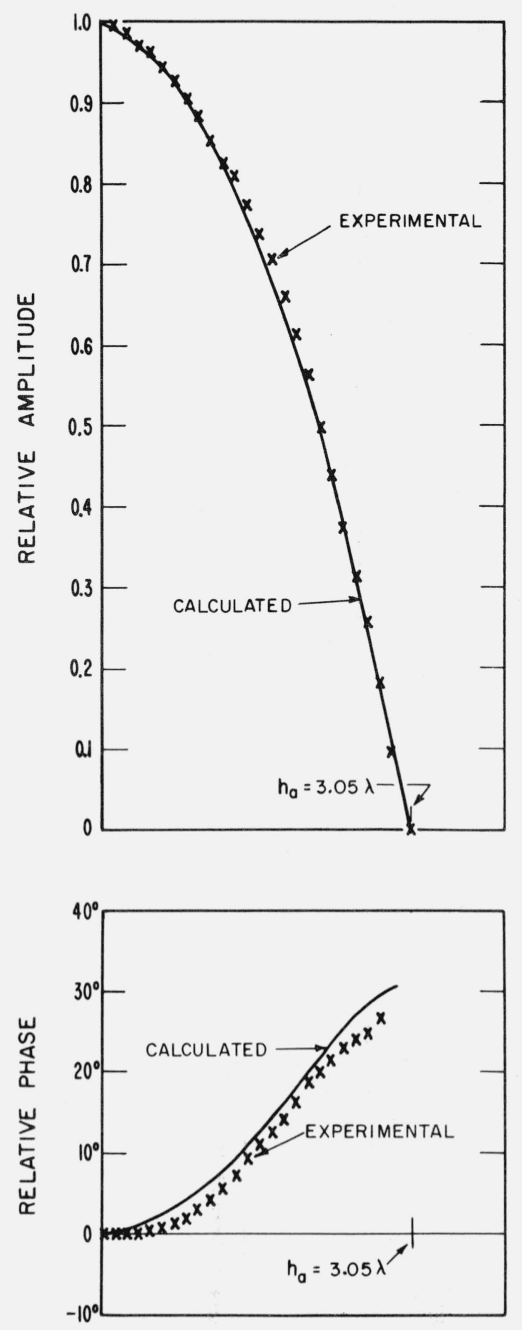

Figure 3. Amplitude and phase variations at the transverse plane $\mathrm{x}=0$ for the case when a $T E_{10}$ mode traveling in the direction of the negative $\mathrm{x}$-axis is launched into port $D$ with $\mathrm{L}=20 \lambda$ (fig. 2).

TABLE 1. Scattering coefficients $\mathrm{T}_{\mathrm{n} 1}^{\mathrm{AD}}$ for the night-to-day propagation path $h_{a}=3.05 \lambda h_{d}=3.18 \lambda L=20 \lambda$

\begin{tabular}{r|r|r}
\hline \hline$n$ & \multicolumn{1}{c|}{$\operatorname{Re}\left(T_{n 1}^{A D}\right)$} & \multicolumn{1}{c}{$\operatorname{Im}\left(T_{n 1}^{A D}\right)$} \\
\hline 1 & $0.1051 \mathrm{E}+01$ & $0.3583 \mathrm{E}-00$ \\
3 & $.1887 \mathrm{E}-01$ & $-.1351 \mathrm{E}-00$ \\
5 & $.7920 \mathrm{E}-04$ & $.1940 \mathrm{E}-01$ \\
7 & $.8953 \mathrm{E}-02$ & $-.2083 \mathrm{E}-02$ \\
9 & $-.2212 \mathrm{E}-02$ & $-.1139 \mathrm{E}-02$ \\
11 & $-.8585 \mathrm{E}-03$ & $-.7392 \mathrm{E}-03$ \\
\hline
\end{tabular}

Where $E \pm X Y=10^{ \pm X Y}$
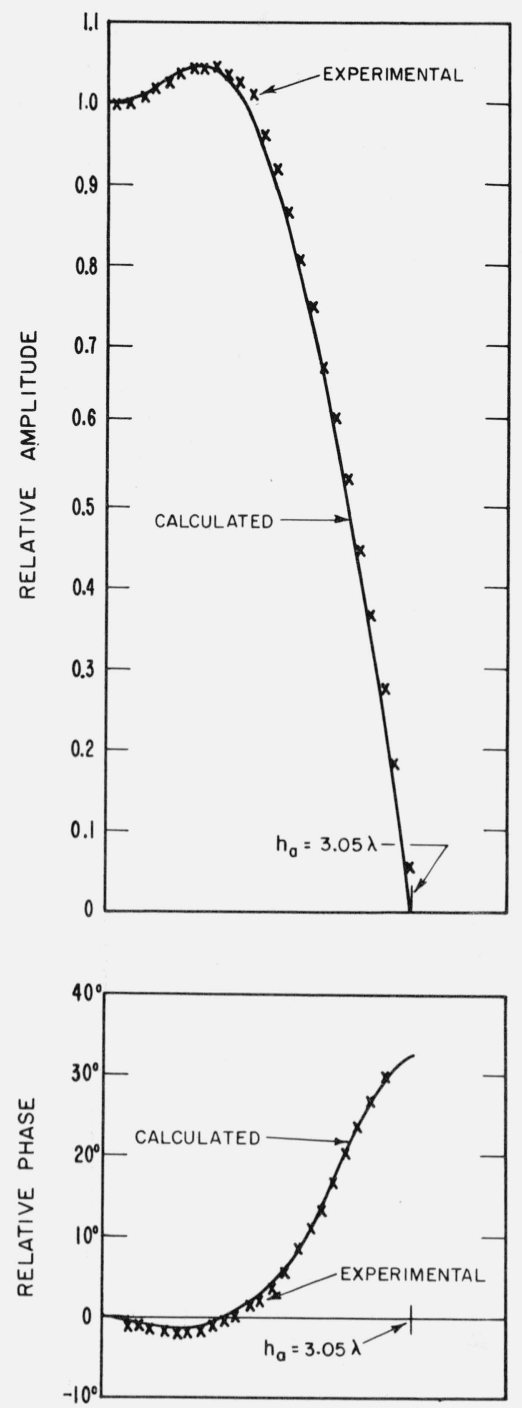

Figure 4. Amplitude and phase variations at the transverse plane $\mathrm{x}=0$ for the case when a $T E_{10}$ mode traveling in the direction of the negative $\mathrm{x}$-axis is launched into port $D$ with $\mathrm{L}=10 \lambda$ (fig. 2).

TABLE 2. Scattering coefficients $\mathrm{T}_{\mathrm{n} 1}^{\mathrm{AD}}$ for the night-to-day propaga. tion path $h_{a}=3.05 \lambda h_{d}=3.81 \lambda L=10 \lambda$

\begin{tabular}{r|r|r}
\hline \hline$n$ & \multicolumn{1}{|c|}{$\operatorname{Re}\left(T_{n 1}^{A D}\right)$} & \multicolumn{1}{c}{$\operatorname{Im}\left(T_{n 1}^{A D}\right)$} \\
\hline & & \\
1 & $0.1090 \mathrm{E}+01$ & $0.1765 \mathrm{E}-00$ \\
3 & $-.1194 \mathrm{E}-00$ & $-.1125 \mathrm{E}-00$ \\
5 & $-.3798 \mathrm{E}-01$ & $.6171 \mathrm{E}-01$ \\
7 & $.1022 \mathrm{E}-02$ & $-.1364 \mathrm{E}-01$ \\
9 & $-.2000 \mathrm{E}-02$ & $.9152 \mathrm{E}-02$ \\
11 & $.1086 \mathrm{E}-02$ & $.5656 \mathrm{E}-03$ \\
\hline
\end{tabular}

Where $E \pm X Y=10^{ \pm X Y}$ 


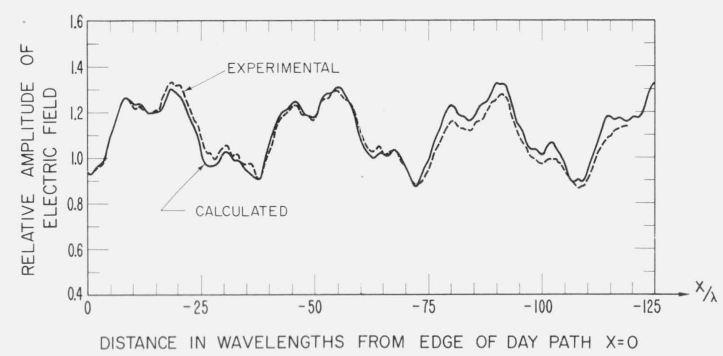

FIGURE 5. Amplitude variations along the negative $\mathrm{x}$-axis for the case when a $\mathrm{TE}_{10}$ mode is launched into port $D$ with $\mathrm{L}=10 \lambda$ (fig. 2).

The first term is clearly the contribution to $T_{31}^{A D}$ due to mode conversion at Junction III (fig. 2) while the second term is due to mode conversion at Junction I.

Now it is interesting to note that, as was predicted by the preceding analysis, the amplitude of the scattering coefficients $S_{31}^{C D}$ and $S_{31}^{A B}$ for $L=20 \lambda$ is half that for the case $L=10 \lambda$. Nevertheless the amplitude of the conversion coefficient $T_{31}^{A D}$ is 12 percent of the amplitude of the transmission coefficient $T_{11}^{A B}$ for the case $L=20 \lambda$ compared with only 13 percent for the case $L=10 \lambda$. It should be noted that this is not due to a large reconversion coefficient from the second mode back into the first mode at Junction I for the case $L=10 \lambda$ since $\left|S_{33}^{A B}\right| \approx 1$ in both cases where $L=10 \lambda$ and $L=20 \lambda$. The behavior of the coefficient $T_{31}^{A D}$ is best understood by noting the phase relationship between the two major contributions to $T_{31}^{A D}$ (i.e., conversion at Junction I and II). While these contributions are only about $10^{\circ}$ out of phase for the case $L=20 \lambda$, they are about $90^{\circ}$ out of phase for $L=10 \lambda$.

The above discussion sheds light on design considerations of a taper section (between two multimode waveguides of different cross sections), with minimum mode conversion. While it has been pointed out in the discussion of the wedge region with the very small gradient that the conversion coefficients decrease as $1 / L$, this is strictly true only if each Junction (fig. 2) is taken separately. On treating the composite junction as one unit it is clear that the total conversion factor does not decrease monotonically with increasing $L$. Thus in order to surpress mode conversion it is necessary to choose the length of the linear taper $L$ such that the two major contributions to $T_{31}^{A D}$ are exactly out of phase and thereby tend to cancel out (destructive interference). This aspect of the problem is not pursued any further in this paper, which is principally concerned with the analysis of the propagation problem.

The amplitude of the electric field along the axis of the waveguide is plotted in figure 5 for the night-to-day propagation path with $L=10 \lambda$. The undulation of the electric field for $x>L$ is clearly seen to be principally due to the interaction of the principal mode with the third and fifth modes.

\section{Concluding Remarks}

It is evident that the quasi-optical technique is applicable to problems of propagation in multimode waveguides when the direction of propagation is from the narrow to the wide waveguide. However, for propagation in the opposite direction it is necessary to modify the quasi-optical approach. In this paper, the required extension has been carried out by applying a generalized reciprocity theorem for waveguide junctions with nonuniform cross sections. Substantiation for this approach is obtained by using a modeling technique which at the same time has provided insight into the nature of the problem.

Finally, it might be mentioned that transmission through a transition region with a gradually varying height profile (not necessarily wedge-shaped) between two multimode waveguides with finite surface impedance boundaries can also be solved by a generalization of the methods described here. This case will be discussed in a subsequent paper by the first author. 
The authors thank C. T. Johnk, S. W. Maley, D. D. Crombie, and A. G. Jean for their encouragement and assistance.

\section{Appendix A. The Generalized Reciprocity Theorem for Waveguide Junctions}

The characteristic admittance for a waveguide of nonuniform cross section is generally not the same for a forward and a backward propagating wave. It is also a function of the distance along the axis of the waveguide. Only in straight cylindrical waveguides with constant cross sections is the characteristic admittance the same for both directions of propagation, and independent of the distance along the axis of the waveguide.

For a radial waveguide, for instance, the characteristic admittance for the principal wave traveling in the direction of increasing radius $\rho$ (Junction II, fig. 2) is [Wait, 1962],

$$
Y_{n}^{+}=-\frac{H_{\Phi}^{+}}{E_{z}^{+}}=i Y \frac{H_{\nu_{n}}^{(2) \prime}(k \rho)}{H_{\nu_{n}}^{(2)}(k \rho)},
$$

where $Y$ is the wave admittance in free space and where $E_{z}^{+}$and $H_{\Phi}^{+}$are the tangential electric and magnetic fields, respectively. Here $H_{\nu_{n}}^{(2)^{\prime}}(u)$ is the derivative of the Hankel function of the second kind and order $\nu_{n}$ with respect to the argument $u$. For perfectly conducting walls the argument of the Hankel functions is

$$
\nu_{n}=\frac{n \pi}{2 \psi}
$$

where $\psi$ is the azimuthal boundary of the radial waveguide as indicated in figure 2 .

The characteristic wave admittance for the wave traveling in the direction of decreasing radius $\rho$ is, on the other hand,

$$
Y_{n}^{-}=\frac{H_{\Phi}^{-}}{E_{z}^{-}}=-i Y \frac{H_{\nu_{n}}^{(1)^{\prime}}(k \rho)}{H_{\nu_{n}}^{(1)}(k \rho)}
$$

where the Hankel function of the first kind replaces the Hankel function of the second kind in (A.1).

It should be noted that $Y_{n}^{+}$is equal to the complex conjugate of $Y_{n}^{-}$, and that for large $k \rho$, $Y_{n}^{+} \simeq Y_{n}^{-} \simeq Y$.

Now, for the region under consideration, it may be shown that [Kerns, 1949]

$$
\int_{S}\left(\bar{E}^{\prime} \times \bar{H}^{\prime \prime}\right)-\left(\bar{E}^{\prime \prime} \times \bar{H}^{\prime}\right) \cdot \bar{n} d S=0
$$

where $S$ is the closed surface bounding the region of integration, $\bar{n}$ is the unit vector normal to $S$, and $\bar{E}^{\prime}, \bar{H}^{\prime}$ and $\bar{E}^{\prime \prime}, \bar{H}^{\prime \prime}$ are any two electromagnetic fields of the same frequency that can exist within the source-free region bounded by $S$. Equation (A.3) is valid in the present context since the electromagnetic characteristics of the medium are isotropic. Furthermore, for the present application, $S$ consists of the inner conducting surface $S_{0}$ of the waveguide junction plus the terminal surfaces $S_{A}$ and $S_{B}$ of the two-port. At the terminal surfaces of the junction, the electric and magnetic fields can be expressed in terms of the basis fields $\Phi_{n}$ (defined in sec. 2), and thus (A.3) may be written as

$$
\int_{S_{A}, S_{B}} \sum_{m} \sum_{n}\left[\Phi_{m}\left(a_{m}^{\prime}+b_{m}^{\prime}\right) \Phi_{n}\left(Y_{n}^{+} a_{n}^{\prime \prime}-Y_{n}^{-} b_{n}^{\prime \prime}\right)-\Phi_{m}\left(a_{m}^{\prime \prime}+b_{m}^{\prime \prime}\right) \Phi_{n}\left(Y_{n}^{+} a_{n}^{\prime}-Y_{n}^{-} b_{n}^{\prime}\right)\right] d S=0
$$


where $a_{m}^{\prime}, b_{m}^{\prime}$ and $a_{m}^{\prime \prime}, b_{m}^{\prime \prime}$ are elements of the total wave amplitude vectors (8), related to the electromagnetic fields $E^{\prime}, H^{\prime}$ and $E^{\prime \prime}, H^{\prime \prime}$, respectively. For waveguide ports with boundaries corresponding to orthogonal surfaces of separable coordinate systems, the basis fields are orthogonal; hence, in this case (A.4) reduces to

$$
\left[\sum_{m}\left(a_{m}^{\prime}+b_{m}^{\prime}\right) W_{m}\left(Y_{m}^{+} a_{m}^{\prime \prime}-Y_{m}^{-} b_{m}^{\prime \prime}\right)-\left(a_{m}^{\prime \prime}+b_{m}^{\prime \prime}\right) W_{m}\left(Y_{m}^{+} a_{m}^{\prime}-Y_{m}^{-} b_{m}^{\prime}\right)\right]=0
$$

where $W_{m}$ is the power normalization coefficient defined in (10). In matrix notation this can be written as

$$
\left(\tilde{a}^{\prime}+\tilde{b}^{\prime}\right) W\left(Y^{+} a^{\prime \prime}-Y^{-} b^{\prime \prime}\right)=\left(\tilde{a}^{\prime \prime}+\tilde{b}^{\prime \prime}\right) W\left(Y^{+} a^{\prime}-Y^{-} b^{\prime}\right)
$$

where $\tilde{a}$ is the transpose of $a$.

Since the transpose of a scalar is equal to itself, (A.6) reduces to

$$
\tilde{a}^{\prime \prime} W Y^{T} S a^{\prime}=\tilde{a}^{\prime \prime} \tilde{\tilde{S}} W Y^{T} S a^{\prime}
$$

where $Y^{T}$, the total characteristic admittance, is defined as

$$
Y^{T} \equiv Y^{+}+Y^{-}
$$

and $b$ is replaced by $S a$, where $S$ is the total scattering matrix defined by (15).

Now since $a^{\prime}$ and $a^{\prime \prime}$ can be chosen arbitrarily, the reciprocity theorem for waveguide junctions is, from (A.7),

$$
W Y^{T} S=\left(\widetilde{W Y^{T} S}\right)
$$

since

$$
\left(\widetilde{W Y^{T}}\right)=W Y^{T}
$$

For completeness, the relationship between the scattering matrix $S$ and the equivalent admittance matrix $\hat{Y}$ of the junction can be shown to be given by

$$
\hat{Y}=\frac{Y^{+}-Y^{-} S}{I+S}, \quad S=\frac{Y^{+}-\hat{Y}}{Y^{-}+\hat{Y}} .
$$

The equivalent admittance matrix $\hat{Y}$ relates the amplitude matrix of the electric field $\hat{E} \equiv a+b$ to the amplitude matrix of the magnetic field $\hat{H} \equiv Y^{+} a-Y^{-} b$ through the equation $\hat{H}=\hat{Y} \hat{E}$. For the case of waveguide ports for which $Y^{+}=Y^{-}$, (A.8) and (A.9) reduce to the more familiar forms.

The total characteristic admittance for a radial waveguide is

$$
Y_{n}^{T}=Y_{n}^{+}+Y_{n}^{-}=\frac{4 Y}{\pi k \rho H_{\nu_{n}}^{(1)}(k \rho) H_{\nu_{n}}^{(2)}(k \rho)}
$$

For waveguides with constant rectangular cross sections, such as port A in section 6 for example, the total characteristic admittance is

$$
Y_{n}^{T A}=2 Y_{n}^{A}=2 Y S_{n}^{A}
$$

where $S_{n}^{A}$ is defined by (12). 


\section{References}

Bain, W. C., R. N. Bracewell, T. W. Straker, and C. H. Westcott (Feb. 1952), The ionospheric propagation of radio waves of trequency $16 \mathrm{kc} / \mathrm{s}$ over distances of about $540 \mathrm{~km}$, Proc. IEE 99, Pt. IV, Monographs 12-54, 250-259.

Crombie, D. D., A. H. Allen, and M. Newman (May 1958), Phase variations of 16 kc/s transmission from Rugby as received in New Zealand, Proc. IEE 105, Pt. B, 301-304.

Bahar, E. (May 5, 1964), Model studies of the influence of ionospheric perturbations on VLF propagation, Technical Report, ARPA, Contract No. CST-7348, Elect. Engr. Dept., University of Colorado, Boulder, Colo.

Bahar, E., and J. R. Wait (1964), Microwave model techniques to study VLF radio propagation in the earth-ionosphere waveguide, Quasi-Optics, ed. Jerome Fox, pp 447-464 (Interscience Publishers, New York, N.Y.).

Kerns, D. M. (May 1949), Basis of the applicatıon of network equatıons to waveguide problems, J. Kes. NBS 42, 515-540, RP1190.

Maley, S. W., and E. Bahar (Jan. 1963), Model studies of the influence of ionospheric perturbations on VLF propagation, Technical Summary Report, ARPA, Contract No. CST-7348, Elect. Engr. Dept., University of Colorado, Boulder, Colo.

Maley, S. W., and E. Bahar (Jan. 1964), Effects of wall perturbations in multimode waveguides, Radio Sci. J. Res. NBS 68D, No. 1, 35-42.

Pierce, J. A. (May 1955), The diurnal carrier phase variations of a 16-kilocycle transatlantic signal, Proc. IRE 43, 584-588.

Solymar, L. (Dec. 1959), Mode conversion in pyramidal-tapered waveguides, Electronic Radio Engr. 36, No. 12, $461-463$.

Wait, J. R. (May 1959), Diurnal change of ionospheric heights deduced from phase velocity measurements at VLF, Proc. IRE 47, No. 5, 998.

Wait, J. R. (Mar--Apr. 1960), Terrestrial propagation of VLF radio waves, J. Res. NBS 64D (Radio Prop.), No. 2, $153-204$.

Wait, J. R. (1961), A comparison between theoretical and experimental data on phase velocity or VLF radio waves, Proc. IRE 49, 1089-1090.

Wait, J. R. (July-August 1962), An analysis of VLF mode propagation for a variable ionospheric height, J. Res. NBS 66D (Radio Prop.), No. 4, 453-461.

Wait, J. R. (1964), Two-dimensional treatment of mode theory of the propagation of VLF radio waves, Radio Sci. J. Res. NBS 68D, No. 1, 81-94.

\section{Additional Related Reference}

Kerns, D. M., and R. W. Beatty (1965), Basic theory of waveguide junctions and introductory microwave network analysis (Pergamon Press Limited, Oxford, England).

(Paper 69D11-578) 